\title{
How to Recommend Preferable Solutions of a User in Interactive Reinforcement Learning?
}

\author{
Tomohiro Yamaguchi ${ }^{1}$, Takuma Nishimura ${ }^{2}$ and Kazuhiro Sato ${ }^{3}$ \\ 1, ${ }^{2}$ Nara National College of Technology, \\ ${ }^{2}$ currently Kyoto University, \\ ${ }^{3}$ FANUC Ltd \\ clopan
}

\section{Introduction}

In field of robot learning (Kaplan et al., 2002), interactive reinforcement learning method in that reward function denoting goal is given interactively has worked to establish the communication between a human and the pet robot AIBO. The main feature of this method is the interactive reward function setup which was fixed and build-in function in the main feature of previous reinforcement learning methods. So the user can sophisticate reinforcement learner's behavior sequences incrementally.

Shaping (Konidaris \& Barto, 2006; Ng et al., 1999) is the theoretical framework of such interactive reinforcement learning methods. Shaping is to accelerate the learning of complex behavior sequences. It guides learning to the main goal by adding shaping reward functions as subgoals. Previous shaping methods (Marthi, 2007; $\mathrm{Ng}$ et al., 1999) have three assumptions on reward functions as following;

1. Main goal is given or known for the designer.

2. Subgoals are assumed as shaping rewards those are generated by potential function to the main goal (Marthi, 2007).

3. Shaping rewards are policy invariant (not affecting the optimal policy of the main goal) (Ng et al., 1999).

However, these assumptions will not be true on interactive reinforcement learning with an end-user. Main reason is that it is not easy to keep these assumptions while the end-user gives rewards for the reinforcement learning agent. It is that the reward function may not be fixed for the learner if an end-user changes his/ her mind or his/ her preference. However, most of previous reinforcement learning methods assumes that the reward function is fixed and the optimal solution is unique, so they will be useless in interactive reinforcement learning with an end-user.

To solve this, it is necessary for the learner to estimate the user's preference and to consider its changes. This paper proposes a new method how to match an end-user's preference solution with the learner's recommended solution. Our method consists of three ideas. First, we assume every-visit-optimality as the optimality criterion of preference for most of endusers. Including this, section 2 describes an overview of interactive reinforcement learning in our research. Second, to cover the end-user's preference changes after the reward function is given by the end-user, interactive LC-learning prepares various policies (Satoh \& 
Yamaguchi, 2006) by generating variations of the reward function under every-visitoptimality. It is described in section 3 . Third, we propose coarse to fine recommendation strategy for guiding the end-user's current preference among various policies in section 4 .

To examine these ideas, we perform the experiment with twenty subjects to evaluate the effectiveness of our method. As the experimental results, first, a majority of subjects prefer each every-visit plan (visiting all goals) than the optimal plan. Second, the majority of them prefer shorter plans, and the minority of them prefer longer plans. We discuss the reason why the end-users' preferences are divided into two groups. These are described in section 5 . In section 6, the search ability of interactive LC-learning in a stochastic domain is evaluated. Section 7 describes relations between our proposed solutions and current research issues on recommendation systems. Finally, section 8 discusses our conclusions and future work.

\section{Interactive reinforcement learning}

This section describes the characteristics on interactive reinforcement learning in our research, and shows the overview of our system.

\subsection{Interactive reinforcement learning with human}

Table1 shows the characteristics on interactive reinforcement learning. In reinforcement learning, an optimal solution is decided by the reward function and the optimality criteria. In standard reinforcement learning, an optimal solution is fixed since both the reward function and the optimality criteria are fixed. On the other hand, in interactive reinforcement learning, an optimal solution may change according to the interactive reward function. Furthermore, in interactive reinforcement learning with human, various optimal solutions will occur since the optimality criteria depend on human's preference.

Then the objective of this research is to recommend preferable solutions of each user. The main problem is how to guide to estimate the user's preference? Our solution consists of two ideas. One is to prepare various solutions by every-visit-optimality (Satoh \& Yamaguchi, 2006), another is the coarse to fine recommendation strategy (Yamaguchi \& Nishimura, 2008).

\begin{tabular}{|c|c|c|c|}
\hline $\begin{array}{c}\text { Type of } \\
\text { reinforcement learning }\end{array}$ & an optimal solution & reward function & optimality criteria \\
\hline standard & fixed & fixed & fixed \\
\hline interactive & may change & interactive & fixed \\
\hline interactive with human & various optimal & may change & human's preference \\
\hline
\end{tabular}

Table 1. Characteristics on interactive reinforcement learning

\subsection{Overview of the plan recommendation system}

Fig. 1 shows an overview of the plan recommendation system. When a user input several goals to visit constantly as his/ her preference goals, they are converted to the set of rewards in the plan recommendation block for the input of interactive LC-learning (Satoh \& Yamaguchi, 2006) block. After various policies are prepared, each policy is output as a round plan for recommendation to the user. The user comes into focus on his/ her preference criteria through the interactive recommendation process. The interactive recommendation will finish after the user decides the preference plan. Next section, interactive LC-Learning block is described. 


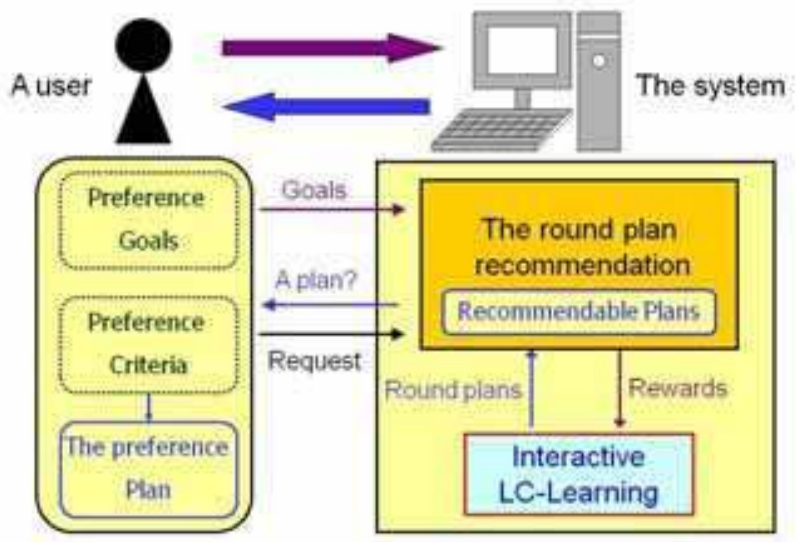

Fig. 1. The plan recommendation system

\subsection{Interactive LC-Learning block}

Fig. 2 shows an overview of interactive LC-Learning (Satoh \& Yamaguchi, 2006) block that is extended model-based reinforcement learning. In Fig. 2, our learning agent consists of three blocks those are model identification block, optimality criterion block and policy search block. The details of these blocks are described in following section. The novelty of our method lies in optimality criterion as every-visit-optimality and the method of policy search collecting various policies.

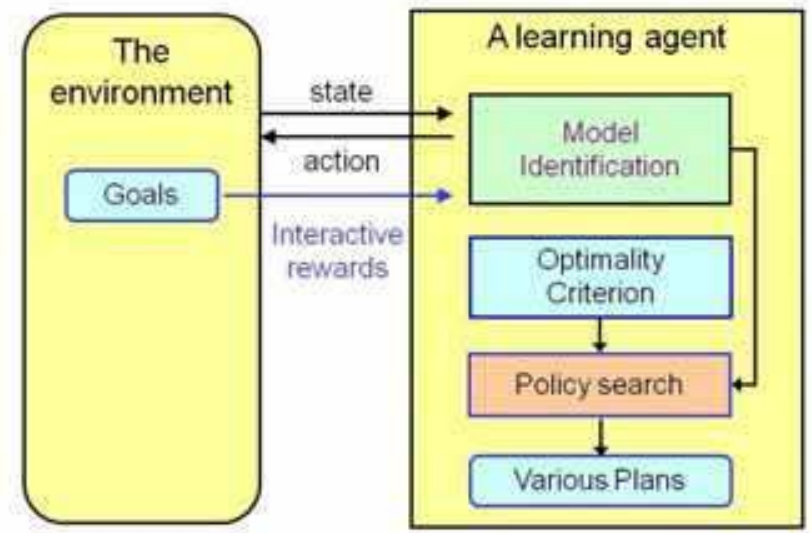

Fig. 2. Interactive LC-Learning block

\subsubsection{Model identification}

In model identification block, the state transition probabilities $P\left(s^{\prime} \mid s, a\right)$ and reward function $R(s, a)$ are estimated incrementally by observing a sequence of $(s, a, r)$. Note that $s$ is an observed state, $a$ is an executed action, and $R w$ is an acquired rew ard. This estimated model is generally assumed Markov Decision Processes (MDP) (Puterman, 2006). MDP model is defined by following four elements. 
1. Set of states: $S=\left\{s_{0}, s_{1}, s_{2}, \cdots, s_{n}\right\}$

2. Set of actions: $A=\left\{a_{0}, a_{1}, a_{2}, \cdots, a_{m}\right\}$

3. State transition probabilities: $P\left(s^{\prime} \mid s, a\right)$ probability of occurring state $s^{\prime}$ when execute action $a$ at state $s$.

4. Reward function: $R(s, a)$ acquired reward when execute action $a$ at state $s$.

\subsubsection{Optimality criterion}

Optimality criterion block defines the optimality of the learning policy. In this research, a policy which maximizes average reward is defined as an optimal policy. Eq. (1) shows the definition of average rew ard.

$$
g^{\pi}(s) \equiv \lim _{N \rightarrow \infty} E\left(\frac{1}{N} \sum_{t=0}^{N-1} r_{t}^{\pi}(s)\right)
$$

where $N$ is the number of step, $r_{\tau}^{\pi}(s)$ is the expected value of reward that an agent acquired at step $t$ where policy is $\pi$ and initial state is $s$ and $E()$ denotes the expected value. To simplify, we use gain-optimality criterion in LC-Learning (Konda et al., 2002a). In that, average reward can be calculated by both the expected length of a reward acquisition cycle and the expected sum of the rewards in the cycle.

Then we introduce every-visit-optimality as the new learning criterion based on average reward. Every-visit-optimal policy is the optimal policy that visits every reward in the reward function. For example, if the reward function has two rewards, the every-visit-optimal policy is the largest average reward one which visits both two rewards. Fig.3 shows the example of an every-visit-optimal policy with two rewards.

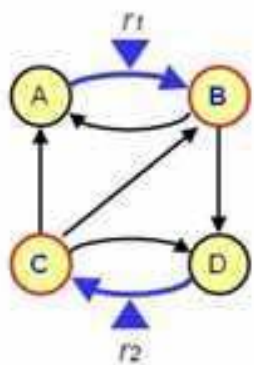

(a) a MDP with two rewards $r_{1}$ and $r_{2}$

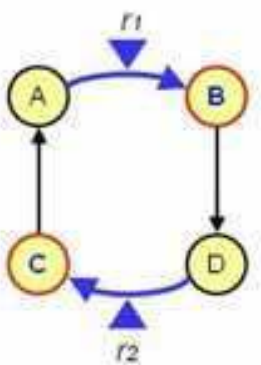

(b) the every-visit-optimal policy

Fig. 3. An Every-visit-optimal policy with two rewards

\subsubsection{Policy search}

Policy search block searches every-visit-optimal policies on an identified model according to optimality of policies. Each policy is converted to a round plan by extracting a cycle. The detail of this block is described in next section.

\section{Preparing various round plans}

This section describes the definition of various round plans and the method for searching various policies. 


\subsection{Illustrated example}

To begin with, we show an illustrated example. Fig.4 shows an overview of preparing various round plans within two rewards. When a MDP has two rewards as shown in Fig.4 (a), then $2^{2}-1$, three kinds of every-visit-optimal policies are prepared (Fig.4 (b)). Each policy is converted to a round plan by extracting a reward acquisition cycle (Fig.4 (c)), since each policy is consists of a rew ard acquisition cycle and some transit passes.

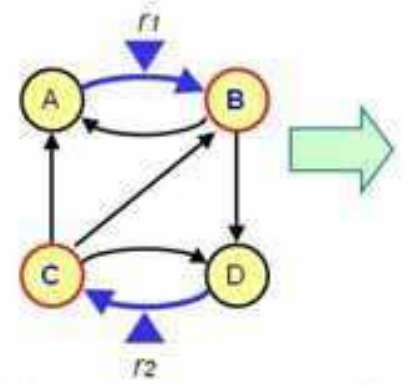

(a) a MDP with two rewards $r_{1}$ and $r_{2}$
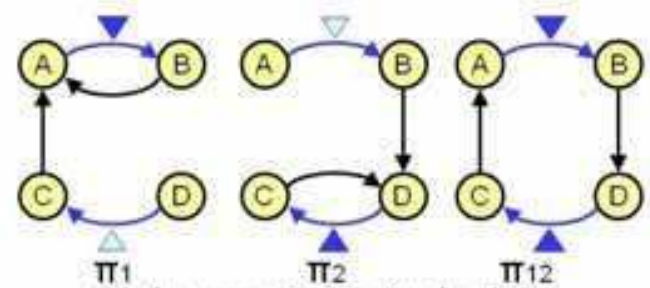

(b) the every-visit-optimal policies

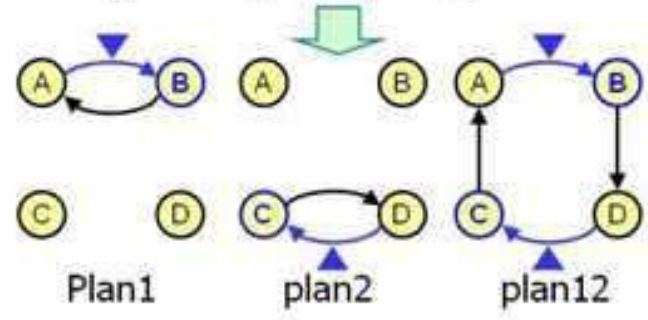

(c) the round plans

Fig. 4. Overview of preparing various round plans

\subsection{Definition of various round plans by every-visit-optimality}

Various round plans are defined by following steps.

1. Enumerate the all subsets of the reward function.

2. Search an every-visit-optimal policy for each subset of the rew ard function.

3. Collect all every-visit-optimal policies and convert them into round plans.

Fig. 5 illustrates the process for searching various round plans. When a reward function is identified as $\{R w 1, R w 2\}$, enumerated subsets of the function are $\{R w 1\},\{R w 2\},\{R w 1, R w 2\}$ in step 1 . Then an every-visit-optimal policy is decided for each subset of the reward function in step 2. At last, these every-visit-optimal policies are collected as various round plans. The number of plans in the various round plans is $2 r-1$, where $r$ is the number of rewards in the model.

\subsection{Searching various policies}

This section describes our various policies search method by interactive LC-Learning (Satoh \& Yamaguchi, 2006). LC-Learning (Konda et al., 2002a; Konda et al., 2002b) is one of the average reward model-based reinforcement learning methods (Mahadevan, 1996). The features of LC-Learning are following;

1. Breadth search of an optimal policy started by each reward rule.

2. Calculating average reward by a reward acquisition cycle of each policy. 


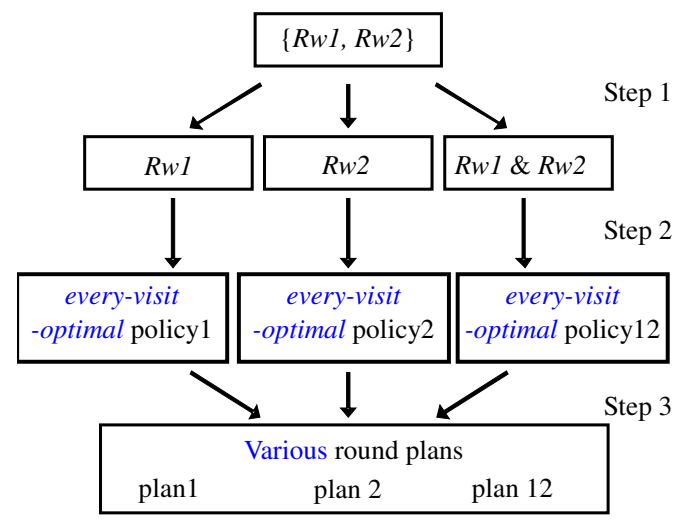

Fig. 5. Process for searching various round plans

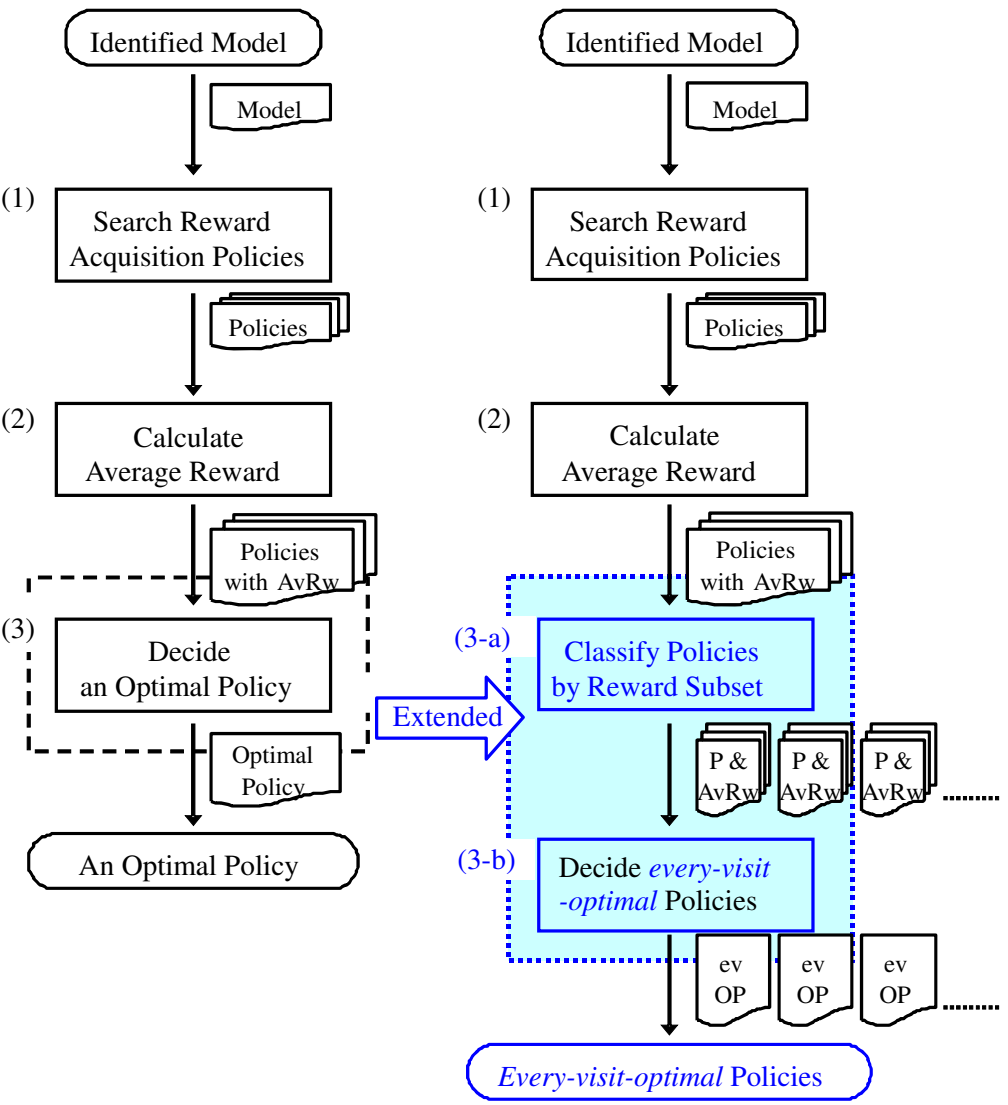

(a) Standard LC- Learning (b) Interactive LC-Learning

Fig. 6. Algorithm for preparing various policies 
Fig. 6 (a) shows standard LC-Learning algorithm. Previous LC-Learning decides an optimalpolicy by following three steps.

1. Search policies that have a rew ard acquisition cycle.

2. Calculate average reward of searched policies.

3. Decide an optimal policy that has the maximum average reward.

Fig. 6 (b) shows algorithm for interactive LC-Learning. Major differences from standard LCLearning are following;

1. Collecting various policies by every-visit-optimality

2. A stochastic version based on occurring probability

3. Adaptable for incremental rew ard addition

Next, we describe the three steps for interactive LC-Learning as shown in Fig.6 (b).

(1) Search rew ard acquisition policies

In this step, reward acquisition policies are searched by converting a MDP into the tree structures where reward acquisition rules are root rule. We show an illustrated example. Fig. 7 shows a MDP model with two rewards $r_{1}$ and $r_{2}$. It is converted into two tree structures. Fig. 8 shows two trees. First, a tree from reward $r_{1}$ as shown in Fig. 8 (a) is generated, then a tree from reward $r_{2}$ as shown in Fig. 8 (b) is generated. In a tree structure, a policy is a path from a root node to the state that is same state to the root node. In a path, an expanded state that is same state to the previous node is pruned since it means a local cycle. In Fig.8, node D and B are pruned states.

Fig. 9 shows all reward acquisition policies in Fig. 7. In a stochastic environment, several rules branch stochastically. In such case, a path from parent node of a stochastic rule to the state that is already extracted is part of a policy that contains the stochastic rule. The policy 12 in Fig. 9 is an example of this.

(2) Calculate average reward

In this step, average reward of each policy is calculated by using occurring probability of each state of the policy. Occurring probability of a state is expected value of the number of transiting the state during the agent transit from the initial state to the initial state. Eq. (2) shows definition of the occurring probability of state $s_{j}$ where initial state is $s_{i}$. Occurring probability of each state is calculated approximately by value iteration using eq. (2).

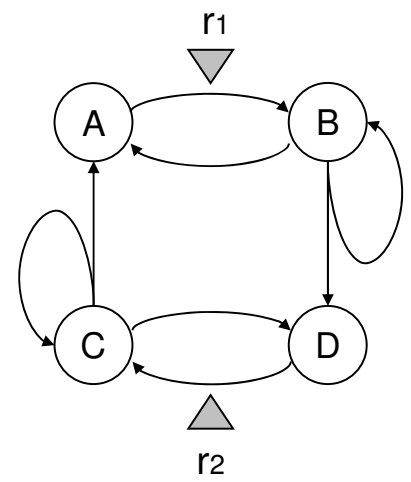

Fig. 7. An example of MDP model 


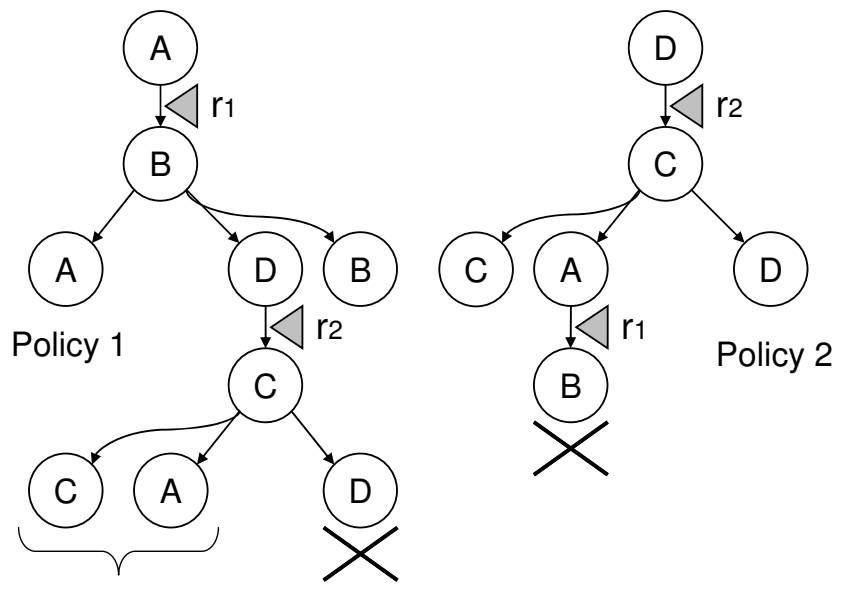

Policy 12

(a) A tree from reward $r_{1}$

(b) A tree from reward $r_{2}$

Fig. 8. Searching rew ard acquisition policies

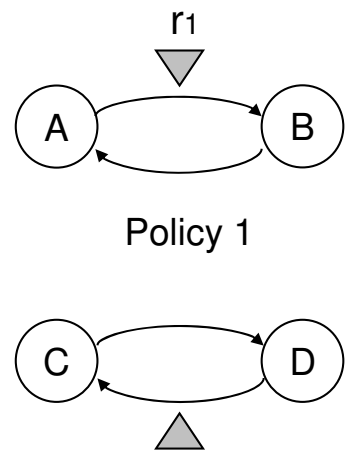

r2

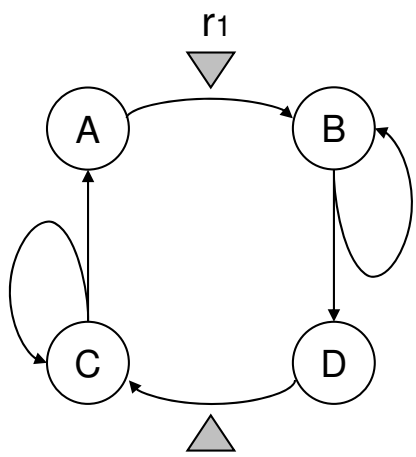

r2

Policy 2

Policy 12

Fig. 9. Three kind of reward acquiring policies

$$
P_{o}\left(s_{j}, s_{i}\right)=\left\{\begin{array}{cc}
1 & (j=i) \\
\sum_{s_{k}} P_{o}\left(s_{k}, s_{i}\right) P\left(s_{j} \mid s_{k}, a_{k}\right) & (j \neq i)
\end{array}\right.
$$

Where $a_{k}$ is the action that is executed at state $s_{k}$.

$$
g^{\pi}\left(s_{i}\right)=\frac{\sum_{s_{j}} P_{o}\left(s_{j}, s_{i}\right) R\left(s_{j}, a_{j}\right)}{\sum_{s_{j}} P_{o}\left(s_{j}, s_{i}\right)}
$$


The average reward of policies is calculated by eq. (3) using occurring probability calculated by eq. (2).

$(3$ '-1) Classify policies by reward subset

In this step, all policies searched by step 1 are classified by acquisition reward set.

(3'-2) Decide every-visit-optimal policies

In this step, an every-visit-optimal policy is decided for each group classified in step (3'-1). Each every-visit-optimal policy is a policy that had maximum average reward in the each group.

\section{Plan recommendation}

This section describes the plan recommendation system and the coarse to fine recommendation strategy (Yamaguchi \& Nishimura, 2008). In this section, a goal is a reward to be acquired, and a plan means a cycle that acquires at least one reward in a policy.

\subsection{Grouping various plans by the visited goals}

After preparing various round plans in section 3.3, they are merged into group by the number of acquired reward. Fig. 10 shows grouping various plans by the number of visited goals. When three goals are input by a user, they are converted into three kinds of reward as $R w 1, R w 2$, and $R w 3$. Then, Group1 in Fig. 10 holds various plans acquiring only one rew ard among $R w 1$, $R w 2$, or $R w 3$. Group2 holds various plans acquiring two kinds of reward among $R w 1$, $R w 2$, or $R w 3$, and Group3 holds various plans acquiring $R w 1$, $R w 2$, and $R w 3$.

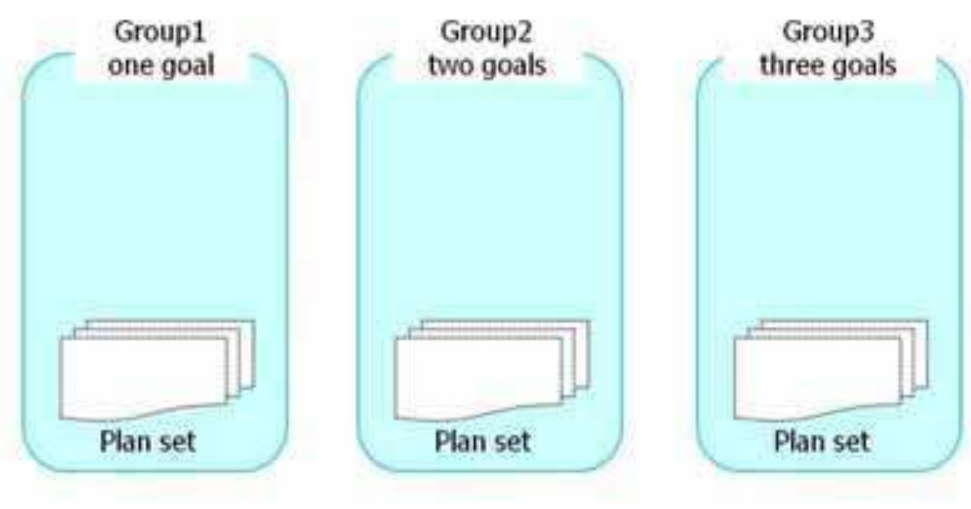

Fig. 10. Grouping various plans

\subsection{Coarse to fine recommendation strategy}

After grouping various plans by the number of visited goals, they are presented to the user sequentially for selecting the most preferable plan. We call the way to decide this order as recommendation strategy. In this paper, we propose coarse to fine recommendation strategy that consists of two steps, coarse recommendation step and fine recommendation step.

(1) Coarse recommendation step

For the user, the aim of this step is to select a preferable group. To support the user's decision, the system recommends a representative plan in each selected group to the user. 
Fig. 11 shows a coarse recommendation sequence when a user changes his/ her preferable group as Group1, Group2, and Group3 sequentially. When the user selects a group, the system presents the representative plan in the group as the recommended plan.

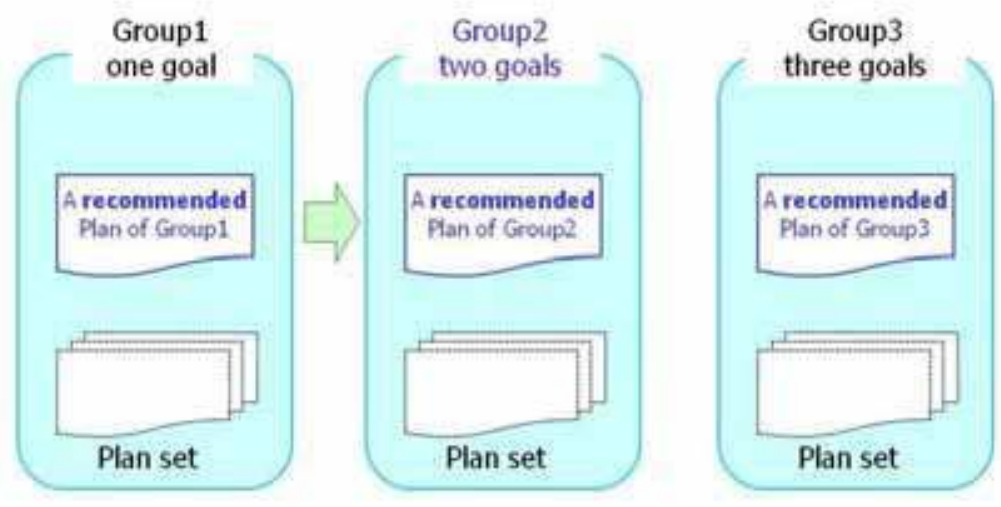

Fig. 11. Coarse recommendation

(2) Fine recommendation step

For the user, the aim of this step is to decide the most preferable plan in the selected group in previous step. To support the user's decision, the system recommends plans among his/ her selected group to the user. Fig. 12 shows a fine recommendation sequence after the user selects his/ her preferable group as Group2. In each group, plans are ordered according to the length of a plan.
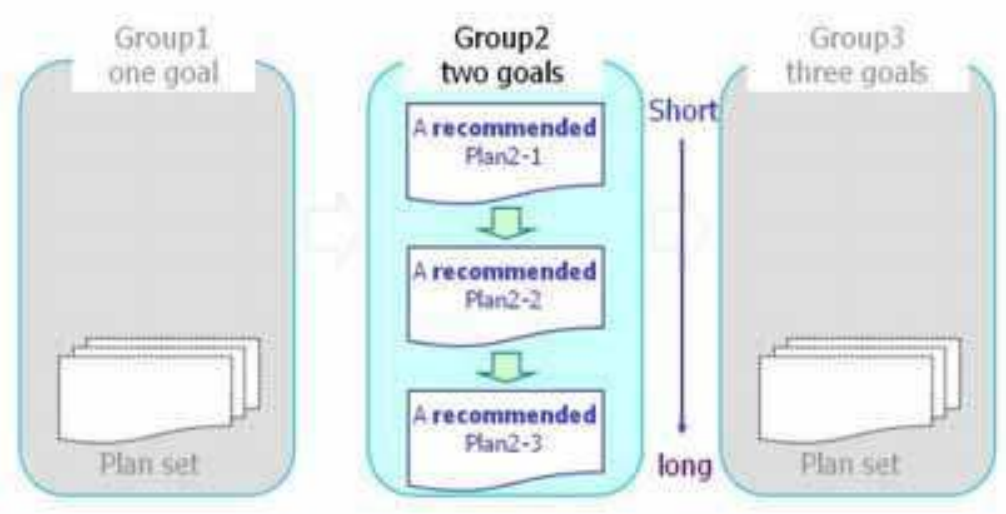

Fig. 12. Fine recommendation in the selected group

\section{Experiment}

We perform the experiment with twenty subjects from 19 to 21 years old to evaluate the effectiveness of our method. 


\subsection{The round-trip plan task}

Fig. 13 shows the round-trip plan Recommendation task in Hokkaido. For a subject, this task is executed by following steps.

1. Each subject selects four cities to visit. Various round-trip plans are recommended.

2. The subject decides the most preferred round-trip plan among them. The task for a subject is to decide the most preferred round-trip plan after selecting four cities to visit among eighteen cities. The task for the system is to estimate the preferable round-trip plans to each user and to recommend them sequentially.

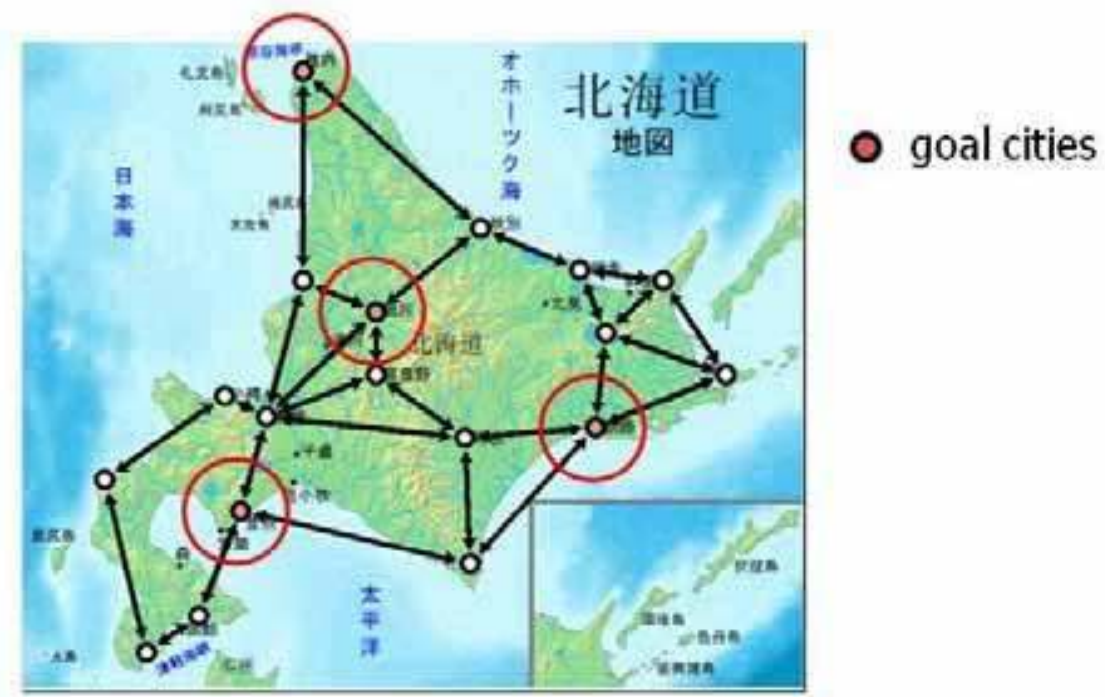

Fig. 13. The round-trip plan Recommendation task

\subsection{Experimental results}

Fig.14 shows the result of the most preferred plans of each twenty subjects. Horizontal axis is the number of visited cities (goals), and vertical axis is the number of subjects. The summary of the experimental result is as follows. First, the majority of subjects prefer each every-visit plan (visit all four cities) than the optimal plan. Second, majority prefers shorter plans, and minority prefers longer plans. Then we focus on these two points.

First point is the effectiveness of every-visit criterion. After selecting four cities, 15 (threequarter) subjects preferred every-visit plans those visit selected four cities. In contrast, only 5 subjects preferred optimal plans with shorter length, yet these plans do not visit all four cities. This suggests that the every-visit criterion is preferable to the optimality criterion for human learners.

Second point is that the users' preferences are divided into two groups, shorter plans, or longer plans. We look more closely the preference for every-visit plans among 15 subjects. Among them, 10 (two-thirds) subjects preferred shorter (every-visit-optimal) plans, and 5 (third) subjects preferred longer (every-visit-non-optimal) plans. Among all 20 subjects, they indicate a similar tendency. Table 2 shows the summary of the experimental result. In table 2 , a majority of subjects prefer shorter plans those are either optimal or every-visit-optimal, a 
minority of subjects prefer longer plans those are every-visit-non-optimal. The reason why the end-users' preferences are divided into two groups will be discussed in the next section.

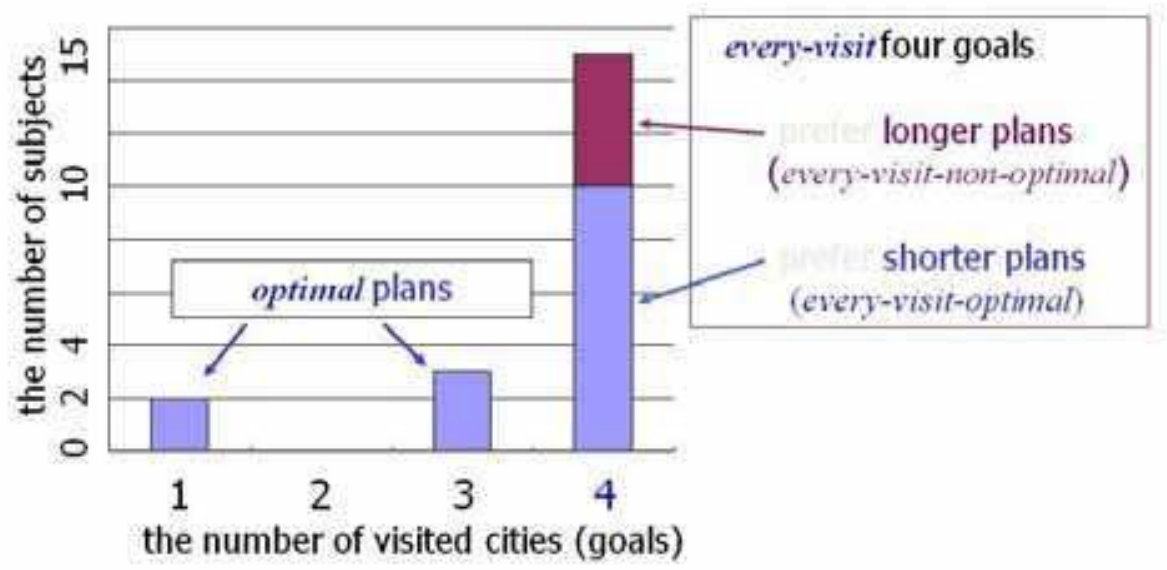

Fig. 14. The result of the most preferred plans

\begin{tabular}{|c|c|c|}
\hline \multirow{2}{*}{ optimal plan } & \multicolumn{2}{|c|}{ every-visit plan } \\
\cline { 2 - 3 } & every-visit-optimal & every-visit-non-optimal \\
\hline short & shorter & long \\
\hline 5 & 10 & 5 \\
\hline
\end{tabular}

Table 2. Summary of the most preferred plans

\subsection{Discussions}

(1) Why the end-users' preferences are divided?

We discuss the reason why the end-users' preferences are divided into two groups. Fig. 15 shows one of the every-visit-optimal plans those major subjects preferred. According to the results of the questionnaire survey, a majority of subjects selected an every-visit-optimal plan have less knowledge on Hokkaido (or no experience to visit Hokkaido).

In contrast, a minority of subjects selected every-visit-non-optimal plans those have additional cities to visit by the plan recommendation. Fig. 16 shows one of the every-visit-non-optimal plans the minority of subjects preferred. According to the results of the questionnaire survey, a majority of subjects selected an every-visit-non-optimal plan have much knowledge or interest on Hokkaido.

It suggests that the preference of a user depends on the degree of the user's background knowledge of the task. In other word, the change of the end-users' preference by the recommendation occurs whether they have the background knowledge of the task or not. Note that in our current plan recommendation system, no background knowledge on the recommended round-trip plan except Fig. 13 is presented to each subject. If any information about recommended plan is provided, we expect that the result on preference change of these two kinds of subjects will differ. 


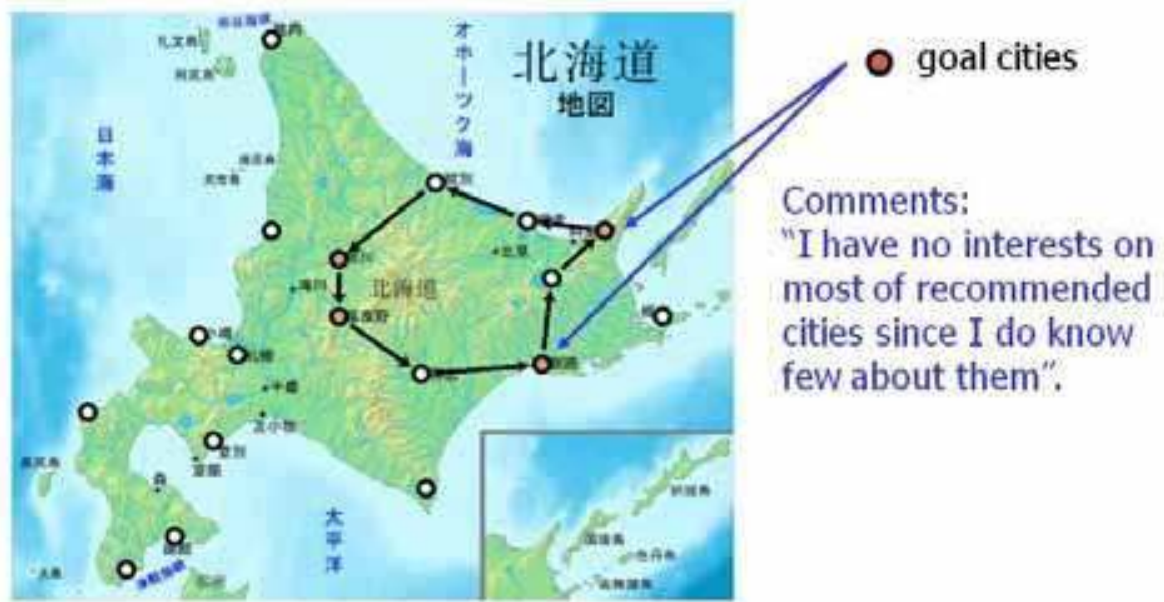

Fig. 15. One of the every-visit-optimal plans

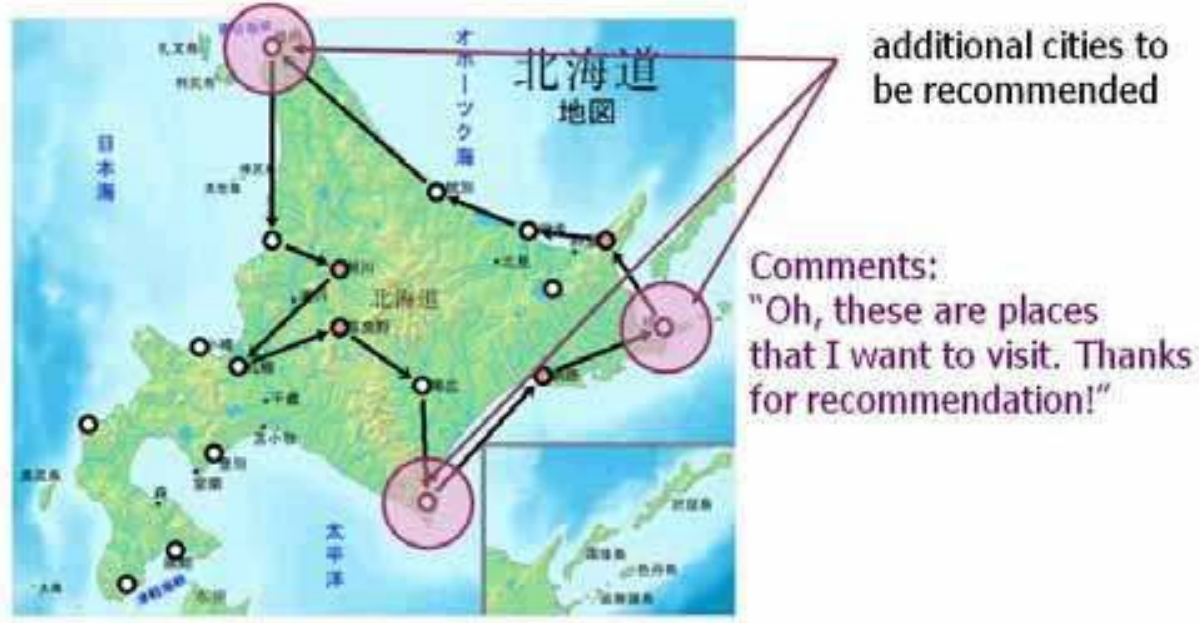

Fig. 16. One of the every-visit-non-optimal plans

(2) The search ability of interactive LC-learning

The computing time of the round-trip plan task in Fig. 13 including graphical output by interactive LC-learning is no more than one second or less per user input, since it is a deterministic MDP model. So we summarize the search ability of LC-Learning in a stochastic case (Satoh \& Yamaguchi, 2006).

We compare two kinds of search abilities of LC-Learning to that of Modified-PIA (Puterman, 2006). First, the search cost of LC-Learning increases linearly when the number of rewards increases linearly. However, the search cost of Modified-PIA increases nonlinearly when the number of rewards increases linearly. Besides, Modified-PIA collects no every-visit optimal policy when the number of rewards is more than three. These suggest that our method is better than previous reinforcement learning methods for interactive 
reinforcement learning in which many rewards are added incrementally. We go into the comparative experiments in detail in section 6.

(3) Every-visit-optimality in a non-deterministic environment

In a stochastic environment, every-visit-optimality is defined as p-every-visit-optimality where each reward is visited stochastically by not less than probability $\mathrm{p}(0<\mathrm{p}=<1)$. It can be calculated by occurring probability of each rewarded rule described in section 3.3 (2). Note that 1-every-visit-optimality is that each reward is visited deterministically even in a stochastic environment.

\section{Evaluating the search ability of interactive LC-learning}

To evaluate the effectiveness of interactive LC-learning in a stochastic domain, comparative experiments with preprocessed Modified-PIA are performed when the number of rewards increases. We compare the two kinds of search abilities as follows.

1. The search cost for every-visit optimal policies

2. The number of collected every-visit-optimal policies

\subsection{Preprocess for Modified-PIA}

Modified-PIA(Puterman, 2006) is one of the model-based reinforcement learning methods based on PIA modified for the average reward. However Modified-PIA is the method to search an optimal policy. So it is not valid to compare the search cost of the Modified-PIA and LC-Learning that searches various policies. To enable to search various policies by Modified-PIA, following preprocess is added. Fig. 17 shows the preprocessed Modified-PIA.

1. Enumerate the models those contain the subset of rew ard set of the original model.

2. Search an optimal policy for each subset of the rew ard function using Modified-PIA.

3. Collect optimal policies.

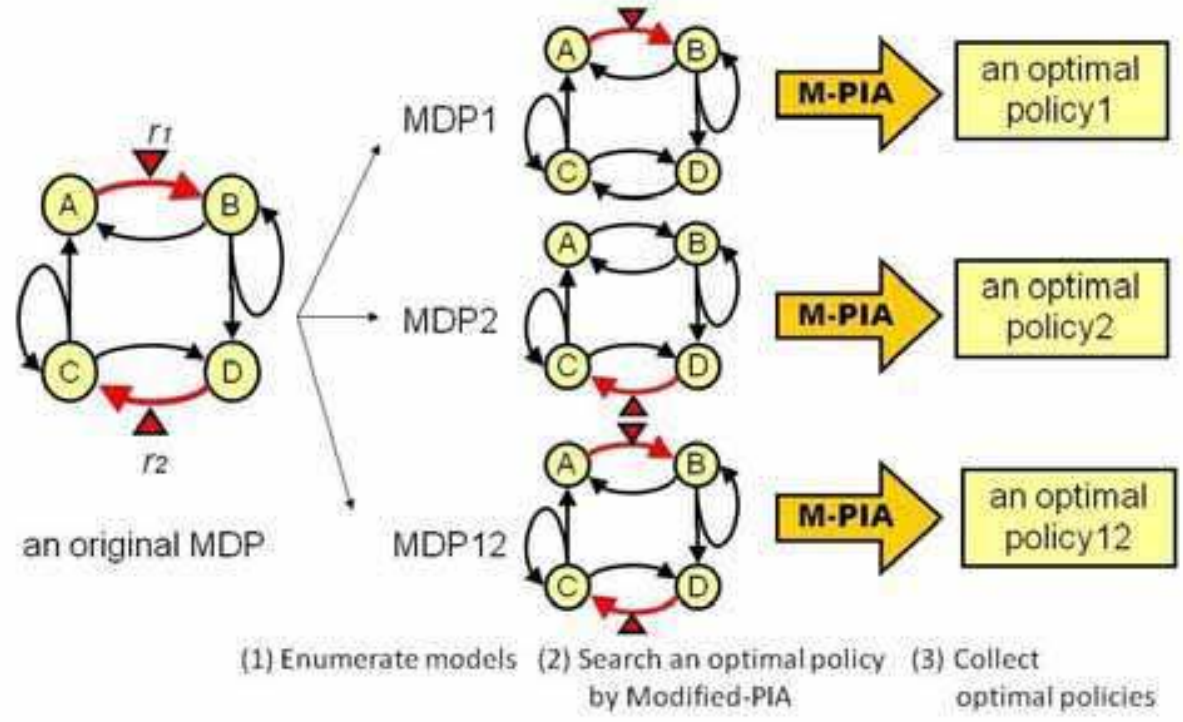

Fig. 17. The preprocessed Modified-PIA 


\subsection{Experimental setup}

We use a hundred of MDP models those consist of randomly set state transition probability and reward function for experimental stochastic environment, in which the number of rewards is varied among 1 to 10 , the number of states is 10 and the number of actions is 4 . As the measure of the search cost, we used the iteration count in calculating the occurring probability of state for LC-Learning and we used the iteration count in calculating the value function for Modified-PIA.

\subsection{The search cost for every-visit-optimal policies}

To begin with, the search cost for every-visit-optimal policies is evaluated. Fig. 18 shows the comparative search cost when the number of rewards increases. The result indicates that the tendency of search cost of LC-Learning is linear and one of Modified-PIA is non-linear when the number of rewards increases.

Then we discuss the theoretical search cost. In Modified-PIA, MDP models those contain the subset of reward set of an original MDP are made and an optimal policy for each MDP is searched. So original Modified-PIA is performed 2r-1 times where $r$ is the number of rewards. After one reward is added, incremental search cost is following.

$$
\left(2^{r+1-1)}-\left(2^{r-1}\right)=2^{r}\right.
$$

Eq. (4) means that the search cost of Modified-PIA increases nonlinearly when the number of rewards increases. In contrast, in LC-Learning, the number of tree structure increase linearly when the number of rewards is increase. So it is considered that the search cost of LC-Learning increase linearly when the number of rew ards increase.

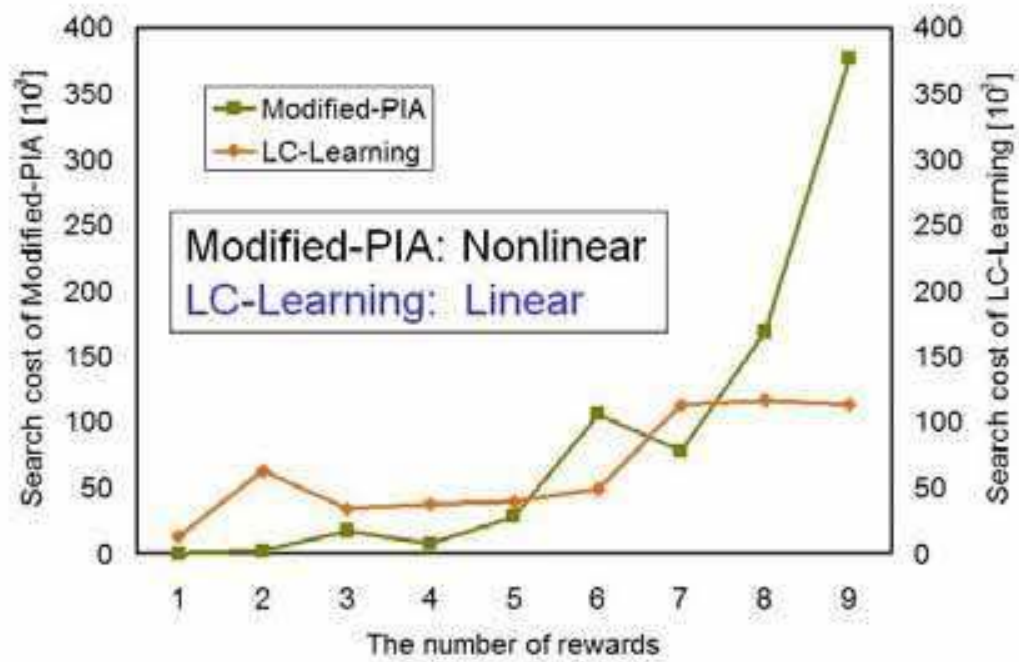

Fig. 18. Search cost when the number of rew ards increases 


\subsection{The number of collected every-visit-optimal policies}

To evaluate the effectiveness of interactive LC-learning, another search ability is compared with preprocessed Modified-PIA. Note that the experimental setup is same as the setup described in section 6.2. Fig. 19 shows the number of collected every-visit-optimal policies. Compared with LC-learning collecting all every-visit-optimal policies, the number of collected every-visit-optimal policies by preprocessed Modified-PIA is smaller than LC-learning.

Then, carefully analyzing the case of six rewards, Fig. 20 shows the rate of collected everyvisit-optimal policies, that is percentage of LC-learning of preprocessed Modified-PIA. It

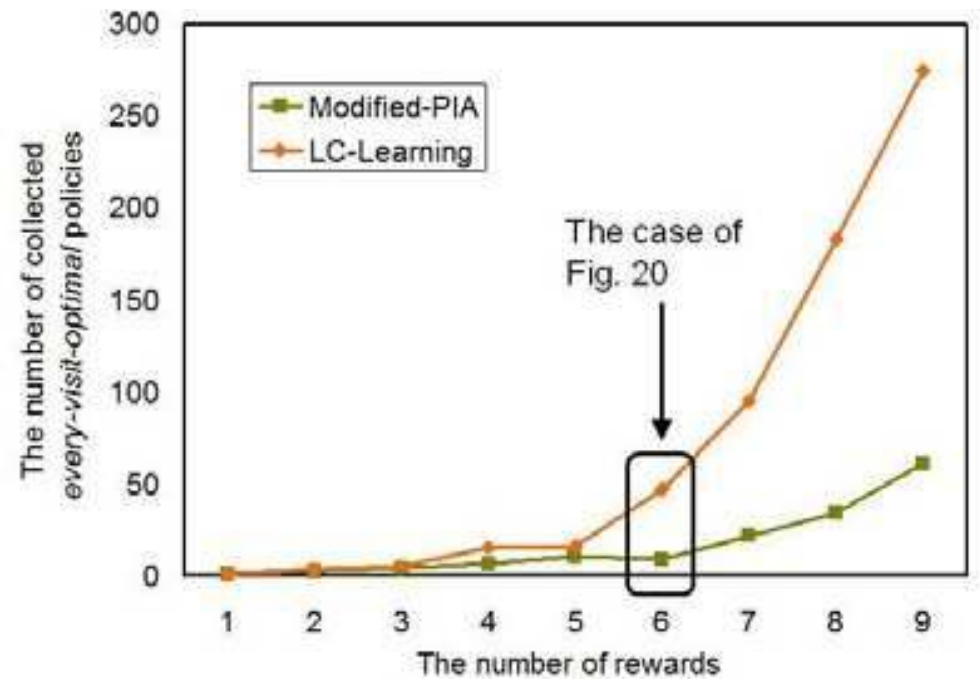

Fig. 19. The number of collected every-visit-optimal policies

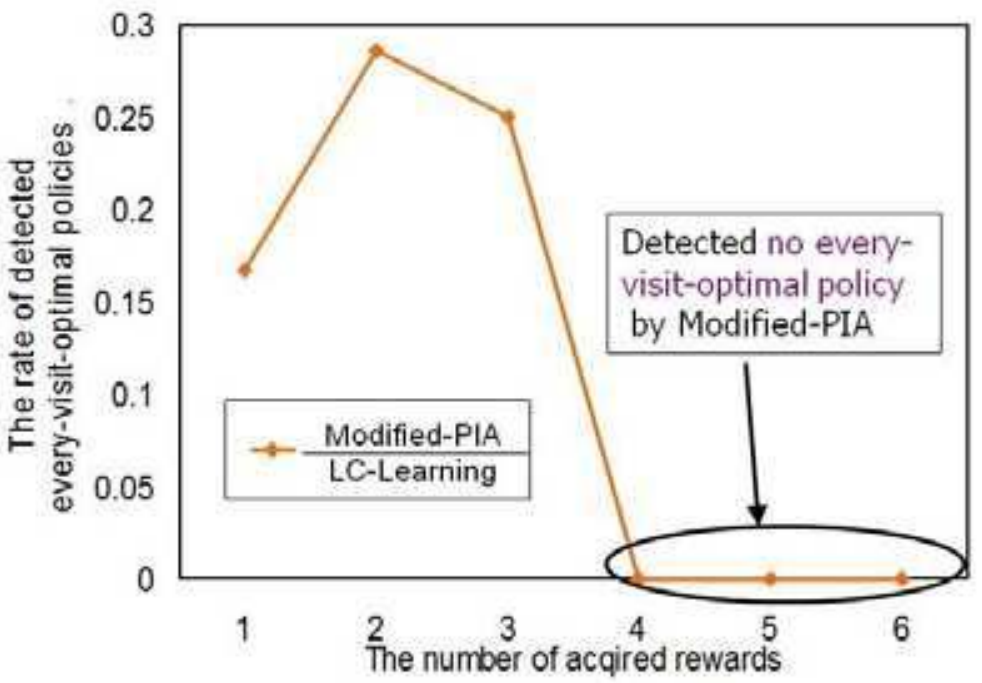

Fig. 20. The rate of collected every-visit-optimal policies 
shows that preprocessed Modified-PIA collects no every-visit-optimal policy when the number of rew ards is more than three.

Then we discuss the reason why the number of collected every-visit-optimal policies by preprocessed Modified-PIA is smaller than LC-learning. Since preprocessed Modified-PIA is based on the standard optimality, it searches an optimal policy in each MDP with the subset of reward set of the original model as shown in Fig.17. It means that preprocessed ModifiedPIA finds an every-visit-optimal policy only if it is same as the optimal policy in each MDP model. As the number of rewards increases, the rate of every-visit-optimal policy that is same as the optimal policy decreases. In other words, the distinction between two criteria becomes larger according to the number of rew ards increases.

Since most previous reinforcement learning methods including Modified-PIA are based on the standard optimality criterion, they only learn an optimal policy. Therefore, under everyvisit-optimality criterion, our method is better than previous reinforcement learning methods for interactive reinforcement learning in which many rewards are added incrementally.

\section{Related works on recommender systems}

This section describes relations between our proposed solutions and current research issues on recommendation systems. The main feature of our recommendation system is interactive and adaptable recommendation for human users by interactive reinforcement learning. First, we describe two major problems on traditional recommenders. Second, interactive recommendation system called Conversational Recommender is summarized. At last, adaptive recommenders with learning ability are described.

\subsection{Major problems on traditional recommenders}

Main objective of recommender systems is to provide people with recommendations of items, they will appreciate based on their past preferences. Major approach is collaborative filtering, whether user-based or item-based (Sarwar et al., 2001) such as by Amazon.com. The common feature is that similarity is computed for users or items, based on their past preferences.

However, there are two major issues. First issue is the similar recommendations problem (Ziegler et al., 2005) in that many recommendations seem to be "similar" with respect to content. It is because of lack of novelty, serendipity (Murakami et al., 2007) and diversity of recommendations. Second issue is the preference change problem (Yamaguchi et al., 2009) that is inability to capture the user's preference change during the recommendation. It often occurs when the user is a beginner or a light user. For the first issue, there are two kinds of previous solutions. One is topic diversification (Ziegler et al., 2005) that is designed to balance and diversify personalized recommendation lists for user's full range of interests in specific topics. Another is visualizing the feature space (Hijikata et al., 2006) for editing a user's profile to search the different items on it by the user. However, these solutions do not directly considering a user's preference change. To solve this, this paper assumes a user's preference change as two-axes space, coarse and fine axes.

\subsection{Interactive recommendation systems}

Traditional recommenders are simple and non-interactive since they only decide which product to recommend to the user. So it is hard to support for recommending more complex 
products such as travel products (Mahmood et al., 2009). Therefore, conversational recommender systems (Bridge et al., 2006) have been proposed to support more natural and interactive processes. Typical interactive recommendation is the following two strategies (Mahmood et al., 2008):

1. Ask the user in detail about her preferences.

2. Propose a set of products to the user and exploit the user feedback to refine future recommendations.

A major limitation of this approach is that there could be a large number of conversational but rigid strategies for a given recommendation task (Mahmood et al., 2008).

\subsection{Adaptive recommenders with learning ability}

There are several adaptive recommenders using reinforcement learning. Most of them observe a user's behavior such as products the user viewed or selected, then learn the user's decision processes or preferences. To improve the rigid strategies for conversational recommenders, learning personalized interaction strategies for conversational recommender systems has been proposed (Mahmood \& Ricci, 2008; Mahmood \& Ricci, 2009; Mahmood et al., 2009).

Major difference from them, the feature of our approach is adaptable recommendation for human users by passive recommendation strategy called coarse to fine recommendation. Adaptable recommendation means that during our recommendation, a user can select these two steps (coarse step or fine step) as his/ her likes before deciding the most preferable plan.

\section{Conclusions}

In this paper, we proposed a new method of interactive LC-learning for recommending preferable solutions of a user.

1. Every-visit-optimality as the optimality criterion of preference for most of end-users was assumed.

2. To cover the end-user's preference changes after the reward function is given by the end-user, interactive LC-learning prepared various policies by generating variations of the reward function under every-visit-optimality.

3. For guiding the end-user's current preference among various policies, coarse to fine recommendation strategy was proposed.

As the experimental results, first, the majority of subjects preferred each every-visit plan (visiting all goals) than the optimal plan. Second, majority preferred shorter plans, and minority prefers longer plans. We discussed the reason why the end-users' preferences are divided into two groups. Then, the search ability of interactive LC-learning in a stochastic domain was evaluated.

The future work is to assist a user for deciding the most preference plan to make his/ herself known the potential preference of the user. To realize this idea, we are evaluating passive recommendation by visualizing the coarse to fine recommendation space and the history of the recommendation of it (Yamaguchi et al., 2009).

\section{References}

Bridge, D., Go"ker, M. H., McGinty, L. \& Smyth, B. (2005). Case-based recommender systems, The Knowledge Engineering Review, Volume 20, Issue 3 (September 2005), pp.315 - 320, Cambridge University Press, ISSN:0269-8889 
Hijikata, Y., Iwahama, K., Takegawa, K., Nishida, S. (2006). Content-based Music Filtering System with Editable User Profile, Proceedings of the 21st Annual ACM Symposium on Applied Computing (ACM SAC 2006), pp.1050-1057, Dijon, France, April, 2006

Kaplan, F.; Oudeyer, P-Y.; Kubinyi, E. \& Miklosi, A. (2002). Robotic clicker training, Robotics and Autonomous Systems, Dillmann, R. et al. (Eds.), pp.197-206, ELSEVIER, ISBN0921-8890, Amsterdam

Konda, T.; Tensyo, S. \& Yamaguchi, T. (2002). LC-Learning: Phased Method for Average Reward Reinforcement Learning - Analysis of Optimal Criteria -, PRICAI2002: Trends in Artificial Intelligence, Lecture notes in Artificial Intelligence 2417, Ishizuka, M. \& Sattar, A. (Eds.), pp.198-207, Springer, ISBN 3-540-44038-0, Berlin

Konda, T.; Tensyo, S. \& Yamaguchi, T. (2002). LC-Learning: Phased Method for Average Reward Reinforcement Learning - Preliminary Results -, PRICAI2002: Trends in Artificial Intelligence, Lecture notes in Artificial Intelligence 2417, Ishizuka, M. \& Sattar, A. (Eds.), pp.208-217, Springer, ISBN 3-540-44038-0, Berlin

Konidaris, G. \& Barto, A. (2006). Automonous Shaping: Knowledge Transfer in Reinforcement Learning, Proceedings of the 23rd International Conference on Machine Learning, pp.489-496, ISBN 1-59593-383-2, Pittsburgh, June, 2006

Mahadevan, S. (1996). Average Rew ard Reinforcement Learning: Foundations, Algorithms, and Empirical Results, Machine Learning, Vol.22, No.1-3, pp.159-195, Springer (Kluwer Academic Publishers), New York

Mahmood, T. \& Ricci, F. (2008). Adapting the interaction state model in conversational recommender systems, Proceedings of the 10th international conference on Electronic commerce, ISBN 978-1-60558-075-3, Innsbruck, August, 2008, ACM, New York

Mahmood, T. \& Ricci, F. (2009). Improving recommender systems with adaptive conversational strategies, Proceedings of the 20th ACM conference on Hypertext and hypermedia, pp.73-82, ISBN 978-1-60558-486-7, Torino, June to July, 2009, ACM, New York

Mahmood, T.; Ricci, F.; Venturini, A. \& Hopken, W. (2008). Adaptive Recommender Systems for Travel Planning, Information and Communication Technologies in Tourism 2008: Proceedings of ENTER 2008 International Conference in Innsbruck, Hopken, W. \& Gretzel, U. (Eds.), pp.1-11, Springer, ISBN 978-3-211-77279-9, New York

Mahmood, T.; Ricci, F. \& Venturini, A. (2009). Improving Recommendation Effectiveness by Adapting the Dialogue Strategy in Online Travel Planning, International eburnal of Information Technology and Tourism, Volume 11, No.4, pp.285-302, ISSN 1098-3058, Cognizant Communication Corporation, New York

Marthi, Bhaskara. (2007). Automatic shaping and decomposition of reward functions, Proceedings of the 24th international conference on Machine learning, pp.601-608, ISBN 978-1-59593-793-3, Corvallis, USA, June, 2007, ACM, New York

Murakami, T.; Mori, K. and Orihara, R. (2007). Metrics for Evaluating the Serendipity of Recommendation Lists, New Frontiers in Artifical Intelligence, Lecture notes in Artificial Intelligence 4914, Satoh, K. et al. (Eds.), pp.40-46, Springer, ISBN 978-3540-78196-7, Berlin

Ng, Andrew Y.; Harada, Daishi; \& Russell, Stuart J. (1999). Policy Invariance Under Reward Transformations: Theory and Application to Reward Shaping, Proceedings of the 16th International Conference on Machine Learning, pp.278-287, Bled, Slovenia, June, 1999 
Preda, M.; Mirea, A.M.; Teodorescu-Mihai, C. \& Preda, D. L. (2009). Adaptive Web Recommendation Systems, Annals of University of Craiova, Math. Comp. Sci. Ser., Vol. 36 (2), pp.25-34

Puterman, M. L. (1994). Markov Decision Processes: Discrete Stochastic Dynamic Programming, JOHN WILEY \& SONS, INC, pp.385-388, ISBN 0471619779, New York

Sarwar, B.; Karypis, G.; Konstan, J. \& Reidl, J. (2001). Item-Based Collaborative Filtering Recommendation Algorithms, Proceedings of the 10th International Conference on World Wide Web, pp.285-295, Hong Kong, May, 2001, ACM, New York

Satoh, K. \& Yamaguchi, T. (2006). Preparing various policies for interactive reinforcement learning, Proceedings of the SICE-ICASE International ebint Conference 2006 (SICEICCAS 2006), pp.2440-2444, Busan, Korea, October, 2006

Yamaguchi \& T., Nishimura, T. (2008): How to recommend preferable solutions of a user in interactive reinforcement learning?, Proceedings of the International Conference on Instrumentation, Control and Information Technology (SICE2008), pp.2050-2055, , Chofu, Japan, August, 2008

Yamaguchi, T.; Nishimura, T. \& Takadama, K. (2009). Awareness based filtering - Toward the Cooperative Learning in Human Agent Interaction -, Proceedings of the ICROSSICE International cbint Conference (ICCAS-SICE 2009), pp.1164-1167, Fukuoka, Japan, August, 2009

Ziegler, C.N.; McNee, S.M.; Konstan, J.A. \& Lausen, G. (2005). Improving Recommendation Lists Through Topic Diversification, Proceedings of the 14th international conference on World Wide Web(WWW2005) , pp.22-32, Chiba, Japan, May, 2005, ACM, New York 


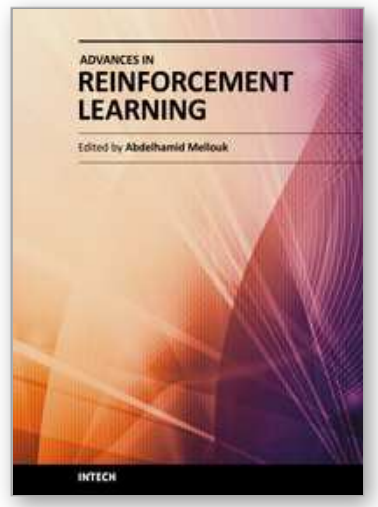

\author{
Advances in Reinforcement Learning \\ Edited by Prof. Abdelhamid Mellouk
}

ISBN 978-953-307-369-9

Hard cover, 470 pages

Publisher InTech

Published online 14, January, 2011

Published in print edition January, 2011

Reinforcement Learning $(R L)$ is a very dynamic area in terms of theory and application. This book brings together many different aspects of the current research on several fields associated to $R L$ which has been growing rapidly, producing a wide variety of learning algorithms for different applications. Based on 24 Chapters, it covers a very broad variety of topics in RL and their application in autonomous systems. A set of chapters in this book provide a general overview of $R L$ while other chapters focus mostly on the applications of RL paradigms: Game Theory, Multi-Agent Theory, Robotic, Networking Technologies, Vehicular Navigation, Medicine and Industrial Logistic.

\title{
How to reference
}

In order to correctly reference this scholarly work, feel free to copy and paste the following:

Tomohiro Yamaguchi, Takuma Nishimura and Kazuhiro Sato (2011). How to Recommend Preferable Solutions of a User in Interactive Reinforcement Learning?, Advances in Reinforcement Learning, Prof. Abdelhamid Mellouk (Ed.), ISBN: 978-953-307-369-9, InTech, Available from: http://www.intechopen.com/books/advancesin-reinforcement-learning/how-to-recommend-preferable-solutions-of-a-user-in-interactive-reinforcementlearning-

\section{INTECH}

open science | open minds

\section{InTech Europe}

University Campus STeP Ri

Slavka Krautzeka 83/A

51000 Rijeka, Croatia

Phone: +385 (51) 770447

Fax: +385 (51) 686166

www.intechopen.com

\section{InTech China}

Unit 405, Office Block, Hotel Equatorial Shanghai

No.65, Yan An Road (West), Shanghai, 200040, China 中国上海市延安西路65号上海国际贵都大饭店办公楼405单元

Phone: +86-21-62489820

Fax: +86-21-62489821 
(C) 2011 The Author(s). Licensee IntechOpen. This chapter is distributed under the terms of the Creative Commons Attribution-NonCommercialShareAlike-3.0 License, which permits use, distribution and reproduction for non-commercial purposes, provided the original is properly cited and derivative works building on this content are distributed under the same license. 